This article was downloaded by:[University of lowa Libraries]

On: 21 July 2007

[University of lowa Libraries]

Access Details: [subscription number 731762562]

Publisher: Taylor \& Francis

Informa Ltd Registered in England and Wales Registered Number: 1072954

Registered office: Mortimer House, 37-41 Mortimer Street, London W1T 3JH, UK

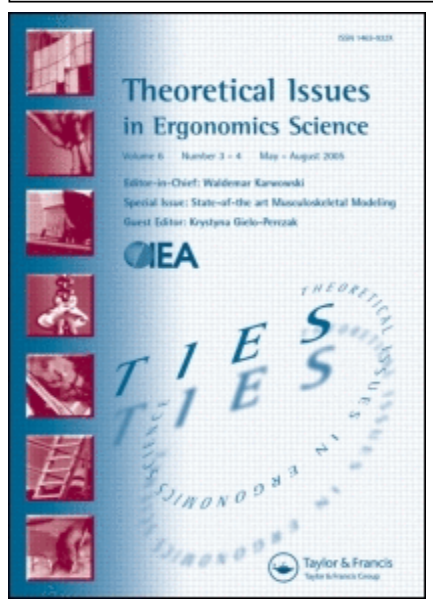

\title{
Theoretical Issues in Ergonomics
} Science

Publication details, including instructions for authors and subscription information: http://www.informaworld.com/smpp/title content=t713697886

Accounting for time-dependent covariates in driving simulator studies

Online Publication Date: 01 January 2007

To cite this Article: Donmez, Birsen, Boyle, Linda Ng and Lee, John D. , (2007)

'Accounting for time-dependent covariates in driving simulator studies', Theoretical

Issues in Ergonomics Science, 1 - 11

To link to this article: DOI: $10.1080 / 14639220701281135$

URL: http://dx.doi.org/10.1080/14639220701281135

\section{PLEASE SCROLL DOWN FOR ARTICLE}

Full terms and conditions of use: http://www.informaworld.com/terms-and-conditions-of-access.pdf

This article maybe used for research, teaching and private study purposes. Any substantial or systematic reproduction, re-distribution, re-selling, loan or sub-licensing, systematic supply or distribution in any form to anyone is expressly forbidden.

The publisher does not give any warranty express or implied or make any representation that the contents will be complete or accurate or up to date. The accuracy of any instructions, formulae and drug doses should be independently verified with primary sources. The publisher shall not be liable for any loss, actions, claims, proceedings, demand or costs or damages whatsoever or howsoever caused arising directly or indirectly in connection with or arising out of the use of this material.

(c) Taylor and Francis 2007 


\title{
Accounting for time-dependent covariates in driving simulator studies
}

\author{
BIRSEN DONMEZ, LINDA NG BOYLE* and JOHN D. LEE \\ Dept. of Mechanical and Industrial Engineering, University of Iowa, \\ 3131 Seamans Center, Iowa City, IA 52242, USA
}

\begin{abstract}
Driving involves multiple cognitive processes that are influenced by a dynamic external environment and internal feedback loops. These processes are typically studied in a simulator environment to capture time-dependent driver performance measures. The primary objective of this research is to show that data analysis techniques that ignore or improperly treat time-dependent covariates will lead to erroneous estimates and conclusions. This is demonstrated with a driving simulator study that was used to test whether a significant decrease in performance occurs in the presence of auditory and visual distractions. A total of 28 drivers participated in a 2 (age) $\times 7$ (strategy) repeated measures experiment. The response variable - accelerator release time - was analysed with and without consideration of time-dependent covariates. Using the inverse headway distance as a time-dependent covariate corrected logically inconsistent results obtained when the covariate was ignored. This indicates that ignoring covariates can actually lead to inappropriate design or policy implications.
\end{abstract}

Keywords: Driving simulator; Time-dependent covariates; Accelerator release; Headway distance; Data analysis

\section{Introduction}

Driving involves a complex sequence of tasks that are influenced by both external environmental conditions and internal feedback loops. Dynamic changes in the driving environment require that the driver responds to these changes. However, current responses are conditioned by prior ones. That is, each time the driver samples the environment, the new response will depend on the driver's previous response. These time-dependent feedback loops are a central feature of human behaviour, but a feature that has often been neglected by researchers because of the experimental and statistical complexity it demands. Even though researchers in statistics widely recognize time-dependent covariates (Faucett et al. 1998, Van Der Laan and Robins 1998, Grilli 2005), only a few human factors and transportation research studies, such as Breckenridge and Dodd (1991) and Bittner et al. (1997) include such analysis.

To capture the time dependency of the interrelated tasks involved in driving, it is necessary to go beyond the traditional psychology experiments that tend to investigate cognitive processes in isolation (Brehmer 2005). Experimental strategies that engage drivers in complex tasks, such as on-road and driving simulator

*Corresponding author. Email: linda-boyle@uiowa.edu 
experiments, allow for the interplay between multiple cognitive processes. For example, maintaining lateral and longitudinal positions on the road demands concurrent acceleration and steering control. The presence of oncoming traffic can increase complexity and influence a driver's ability to maintain the necessary longitudinal control. A specific consequence is that, in addition to the time dependencies, dependent measures will also have interrelations when they are viewed at a cross section in time. Concentrating on only one dependent measure can potentially discount many other correlated factors. Therefore, the interrelationships due to the complexity and dynamic nature of the environment and cognitive processes merit consideration.

Because driving experiments closely replicate driving behaviour, they generate interrelated covariates. Such covariates come in two types: fixed over time; and time dependent. For example, baseline measurements, which are subject-specific, can be treated as covariates that are fixed over time. Studies investigating alcohol, fatigue, workload and distraction effects on driver state typically use physiological responses, such as electroencephalogram, electrocardiogram and galvanic skin response (Brookhuis 1998, Collet et al. 2003). These physiological responses require a baseline measurement, which remains unchanged or fixed over the time of the experiment. This measure is then used as a covariate to observe changes over the different treatment groups. Time-dependent covariates, on the other hand, change each time a measurement is taken on a response variable. These covariates may be purely environmental factors, such as changing visibility or traffic congestion, or can reflect the driver's previous response. Ignoring the time-dependency can result in misleading parameter estimates and erroneous conclusions. This study demonstrates that ignoring time dependency between responses can affect both the results and conclusions.

It can be quite challenging to develop a study to understand the factors affecting drivers' behaviour. If the study is too simplistic, it could be argued that the study does not adequately emulate a driving environment; if it is too complicated, there may not be enough control over the independent variables to discern meaningful results (Chapanis 1988, Liu et al. 1999). Simulator experiments lie on a continuum of complexity between traditional psychology experiments and naturalistic on-road studies. Driving simulators provide a quasi-controlled venue that engages multiple cognitive processes such that the output of these interacting processes can be studied. Driving simulators are widely used in human factors and transportation research to explore issues in driving behaviour, in part because of this capability. For example, driving simulators have been used to assess driving performance given medical impairments (Findley et al. 1989, Rizzo et al. 2001), age effects (Brouwer et al. 1991, Syzlek et al. 1995) and the use of new transportation systems ranging from roadway infrastructure to in-vehicle systems (Stanton et al. 2001, Boyle and Mannering 2004). All of these applications balance the need for realistic complexity and control. Time-dependent covariates help quantify the behaviour that occurs in these situations.

The effects of time-dependent covariates are demonstrated with a driving simulator study that was initially conducted to assess the effects of distractions, as well as of different feedback mechanisms to mitigate these effects (Donmez et al. 2006). The following section contains a description of the driving simulator experiment, the response variable (accelerator release time) and the time-dependent covariate (inverse headway distance). The results of the analysis with and without the 
time-dependent covariate are then presented, followed by a discussion of the implications of these findings.

\section{Method}

Driving simulator experiments generate time-dependent covariates, such as vehicle headway distances, that are different for each lead vehicle braking event. Within each condition, there usually are several braking events, thus warranting the need for time-dependent covariates.

The data analysed in this study are from a driving simulator experiment that is representative of other studies done in this area (Lee et al. 2001, Jamson et al. 2004) and that was conducted in part to assess the effects of driver distractions and different strategies to help mitigate them. For a complete description of the experiment, see Donmez et al. (2006).

\subsection{Participants}

A total of 16 middle-aged (range: 35 to $55 ; \bar{X}=45$, s: 17.1 ) and 12 older drivers (range: 65 to $75 ; \bar{X}=69$, s: 11.3 ) completed the study.

\subsection{Apparatus}

The experiment was conducted with a medium-fidelity, fixed-based simulator powered by the DriveSafety ${ }^{\mathrm{TM}}$ Research Simulator (GlobalSim, Inc.), a fully integrated, high-performance driving simulation system designed for use in ground vehicle research and training applications.

\subsection{Experimental design}

The experiment was a $2 \times 7$ repeated measure design with age as a between-subject variable (two levels), and strategy as a within-subject variable (seven levels). Strategy included seven levels: no-distraction, auditory distraction, visual distraction and four different feedback types. In the visual and auditory distraction conditions, the participants were engaged in distracting activities that required them to direct visual attention away from the roadway or to listen to auditory messages, respectively. For the no-distraction condition, participants drove without distractions. For the feedback conditions, participants received feedback based on roadway events. The four different feedback types were auditory-advising, auditory-locking, visual-advising and visual-locking. The results regarding the feedback effects are presented in detail in Donmez et al. (2006).

\subsection{Response variable: accelerator release time}

The participants were asked to follow a lead vehicle that braked periodically. This is a common test scenario used in driving simulator experiments, with a key 


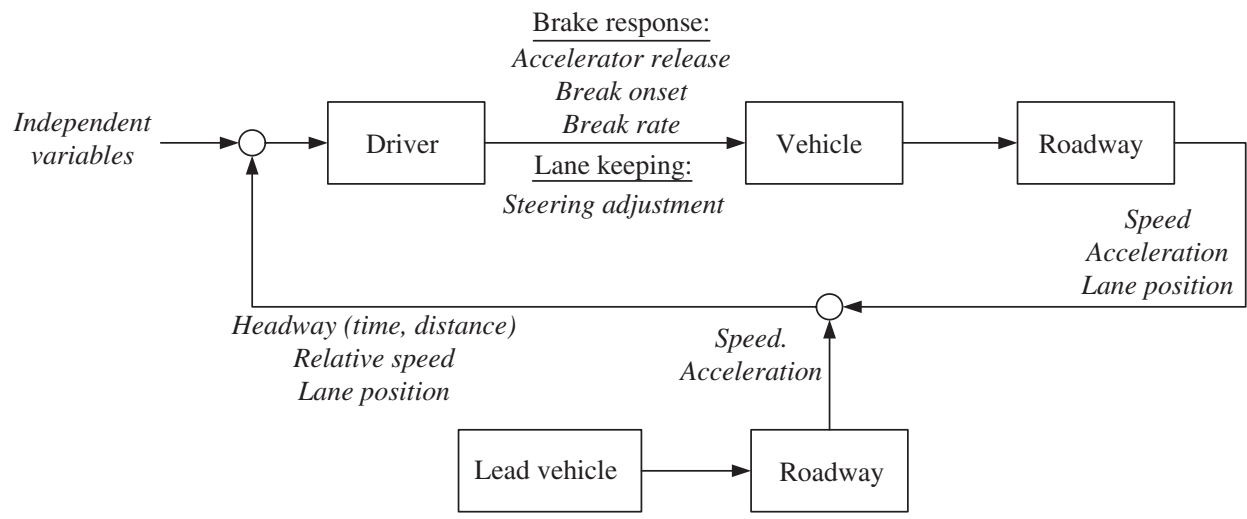

Figure 1. Conceptual control theory model for driving.

performance measure being brake response time (Lee et al. 2001, Jamson et al. 2004). Brake response time is defined as the time from the lead vehicle braking to the release of the accelerator, and the transition time from the accelerator to the brake. The period between the lead vehicle braking and when the driver releases the accelerator is the accelerator release time, which is used as the response variable in this study. Compared to other measures, accelerator release time is a more direct measure of initial reaction because the reactions that follow accelerator release, such as transition time from the accelerator to brake pedal, are dependent on this initial reaction. Accelerator release time is influenced by the vehicle headway distance and the magnitude of cues, such as the rate of change in the visual angle (defined below), thus defining a critical driver feedback loop. It is therefore important to recognize that driving is a closed-loop response (figure 1). That is, each response from the driver creates a new driving situation to which the driver has to react.

\subsection{Time dependent covariate: inverse headway distance}

In a truly controlled experiment, velocities of, and the distance between the vehicles would be controlled. However, such a high level of control results in a very artificial setting that fundamentally changes the driver's role by removing the speed maintenance task. In order to generate a realistic scenario that maintains a degree of experimental control, researchers generally prefer to force a constant headway time to the vehicle ahead. This ensures that repeated braking events are similar in at least one respect. The approach still does not allow for complete control, as participant speed determines the distance between vehicles. As a result, even though the experimenters precisely control the time headway of the lead vehicle at the start of the braking event, the situation can be different for every repetition and can affect the response.

In this simulator experiment, the lead vehicle speed was smoothly adjusted to maintain a constant headway time of 1.8 seconds before all lead vehicle braking events; headway distance could thus vary depending on a driver's speed. Headway distance is therefore a dependent variable but it can also be considered as a covariate, since it may affect the braking response. Inverse headway distance (i.e. the reciprocal of the headway distance) at the onset of the lead vehicle braking was used as a 


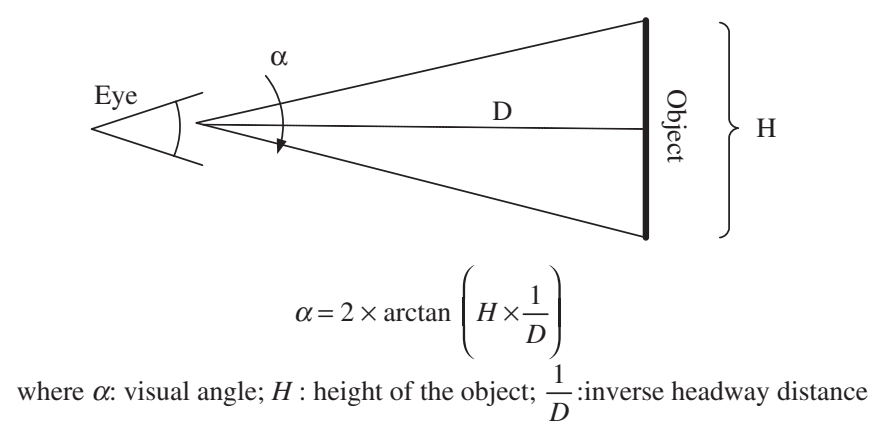

Figure 2. Visual angle.

covariate because this variable defines the visual angle when the lead vehicle brakes (figure 2). A critical cue that guides braking is the rate of expansion of the visual angle of the lead vehicle, which is a function of vehicle size, distance and relative velocity (Lee 1976). In this experiment, the height was constant since there was only one lead vehicle and only the headway distance varied. Moreover, for small angles, the tangent of the angle can be approximated as the value of the angle itself. Therefore, inverse headway distance could be used as the covariate replacing visual angle. Smaller inverse headway distance, i.e. longer distance between vehicles, will result in a smaller visual angle and in a less salient braking event.

\subsection{Data analysis}

Two analyses of accelerator release time were performed. In the first analysis, the inverse headway distance, the time-dependent variable, was ignored. The second analysis included the inverse headway distance as a covariate. The time-dependent covariate analysis was based on a model defined by Rao (1995), which is a special case of a general mixed linear model for repeated measures design (Khattree and Naik 1999). The analyses were conducted using standard general linear mixed modelling techniques and PROC MIXED in SAS 9.1 (SAS Institute, Cary, NC, USA). When there were missing covariate values at any time for a particular subject, the corresponding value of the dependent variable was also discarded (Khattree and Naik 1999). For repeated measures, a compound symmetry covariance structure was chosen based on Schwarz Bayesian Criterion (Schwarz 1978). F tests on main and interaction effects and pair-wise comparisons between the levels of significant main and interaction terms were performed.

\section{Results}

Generally, the results show that without considering the covariate, distractions appear to improve driver performance, whereas when the covariate is included distractions have detrimental effects. 
Table 1. The overall statistical significance for accelerator release time with and without inverse headway distance as a covariate.

\begin{tabular}{|c|c|c|c|c|}
\hline & \multicolumn{2}{|c|}{ Covariate omitted } & \multicolumn{2}{|c|}{ Covariate included } \\
\hline & $F$-value & $p$-value & $F$-value & $p$-value \\
\hline Age & $F(1,26)=1.53$ & NS & $F(1,26)=0.43$ & NS \\
\hline Strategy & $F(6,138)=2.97$ & $<0.01$ & $F(6,138)=2.67$ & $<0.05$ \\
\hline Age $\times$ Strategy & $F(6,138)=0.64$ & NS & $F(6,138)=1.59$ & NS \\
\hline Covariate: Inverse headway distance & & & $F(1,144)=73.92$ & $<0.001$ \\
\hline Age $\times$ Inverse headway distance & & & $F(6,144)=0.05$ & NS \\
\hline Strategy $\times$ Inverse headway distance & & & $F(6,144)=2.95$ & $<0.01$ \\
\hline Age $\times$ Strategy $\times$ Inverse headway distance & & & $F(6,144)=1.39$ & NS \\
\hline
\end{tabular}

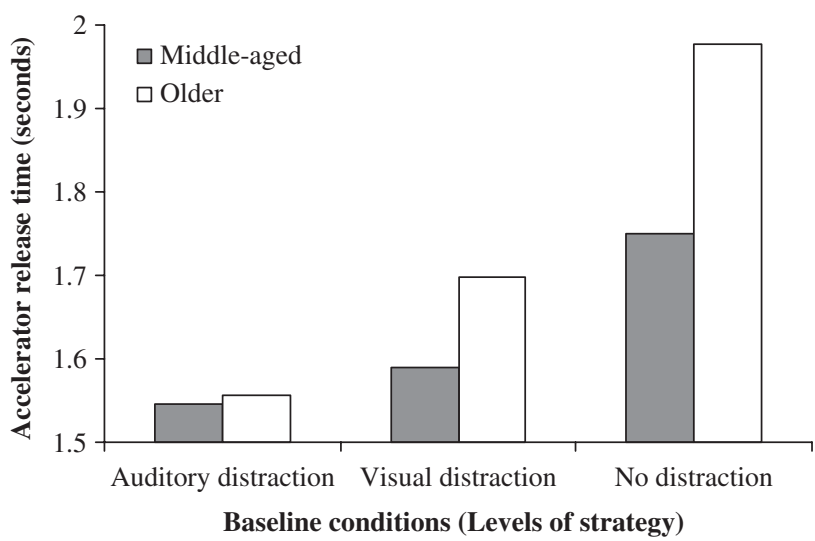

Figure 3. Accelerator release time for distracted and non-distracted conditions (means from raw data).

Without the covariate, strategy had a significant effect on accelerator release time analysis, as shown in table 1 . The plot of the three levels of this effect would suggest that distractions may result in faster accelerator release and therefore in better driving performance (figure 3 ). The results indeed showed that auditory distractions generate a faster response than the non-distracted condition (auditory distraction vs. no distraction: $t(138)=-2.08, p<0.05$, mean difference -0.328 seconds $(95 \% \mathrm{CI}$ $-0.640,-0.017)$; visual distraction vs. no distraction: $t(138)=-0.23, p>0.05$, -0.232 seconds (CI $-0.545,0.082)$ ). However, these results conflict with welldocumented results that show that distractions degrade driving performance (Brown et al. 1969, Alm and Nilsson 1995, Cooper and Zheng 2002, Lam 2002).

Based on the feedback loop between accelerator release time and the strength of the stimulus provided to the driver, as measured by inverse headway distance at the onset of the lead vehicle braking, the relationship between distractions and inverse headway distance was explored. The analysis showed a significant difference for inverse headway, indicating that this may have been a confounding variable and thus it may provide a possible explanation for the counterintuitive trend in the accelerator release time data. The results show that strategy had 


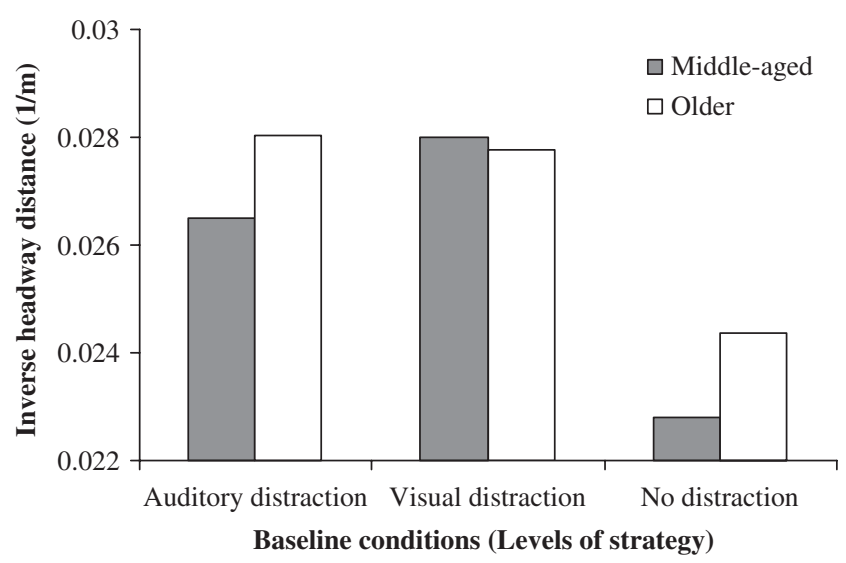

Figure 4. Inverse headway distance for distracted and non-distracted conditions (means from raw data).

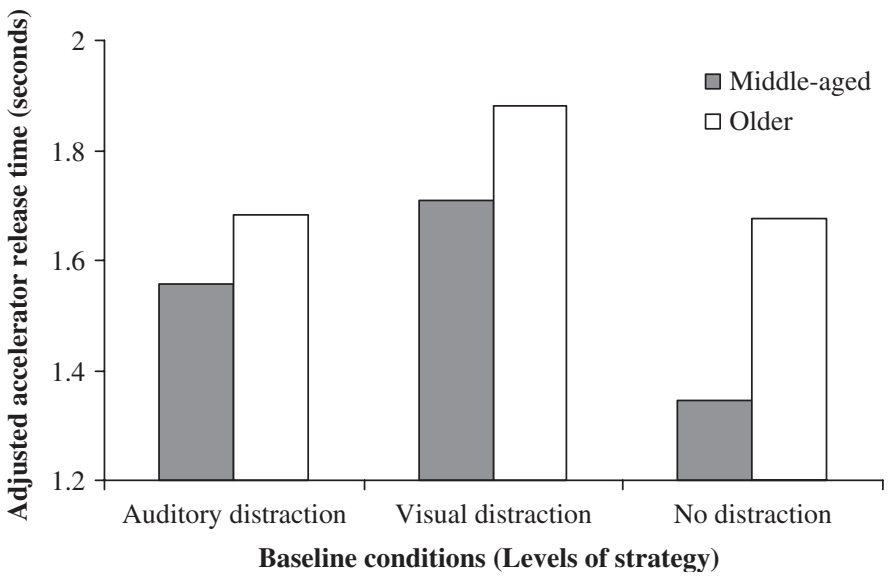

Figure 5. Adjusted accelerator release times with inverse headway distance as a covariate.

a significant effect on inverse headway distance $(F(6,138)=6.73, p<0.0001)$ (figure 4). Non-distracted drivers had lower inverse headway distance values and therefore had smaller visual angles and less salient events (auditory distraction vs. no distraction: $t(138)=-3.99, p<0.05,-0.004 \mathrm{~m}^{-1}$ (CI -0.006 , $-0.002)$; visual distraction vs. no distraction: $t(138)=-4.57, \quad p<0.05$, $-0.005 \mathrm{~m}^{-1}$ (CI $\left.-0.007,-0.003\right)$ ).

When inverse headway distance is added to the model as a covariate, the trend in the results is quite different than when the covariate was ignored (figure 5). The covariate had a negative coefficient estimate $(t(144)=-2.50, p<0.05$, estimate: -62.7); therefore, accelerator release time and inverse headway distance are inversely related. That is, a long headway is associated with a long accelerator release time. As expected, visual distractions resulted in longer accelerator release times when the covariate was taken into account (visual distraction vs. no distraction: $t(138)=2.16$, $p<0.05,0.286$ seconds (CI $0.025,0.547)$ ) and auditory distractions did not have 
a significant effect on reaction time (auditory distraction vs. no distraction: $t(138)=0.85, p>0.05,0.111$ seconds $(\mathrm{CI}-0.147,0.369))$.

\section{Discussion}

The complexity of the driving environment requires the driver to respond to multiple inputs over time. These inputs include approaching curves, speed limits, lead vehicles, oncoming traffic, etc. In addition to requiring the driver to respond to multiple inputs, the driving task at any given time is also a closed loop response. That is, the driver's response changes the road environment and this information is available to the driver as feedback that influences subsequent responses. The multiple inputs associated with the driving task, as well as its closed loop nature, results in dependent measures that are interrelated. These dependencies should be considered in the analysis of driving data.

The interdependencies between measures can be accounted for with timedependent covariates. Neglecting these covariates may lead to inappropriate conclusions, which may in turn lead to improper design guidelines and policies. This paper provides a case study in which the results from a driving simulator study dramatically change with the inclusion of a time-dependent covariate. When the covariate is not included, results show that distractions enhance driver performance by generating short accelerator release times in response to a lead vehicle braking event. The experiment was set up to control for the time headway. This then led drivers to compensate for the distraction by reducing their speed, resulting in a closer lead vehicle and a more salient braking event. This salience resulted in a fast accelerator release. When the analysis included a time-dependent covariate describing this phenomenon, results suggest that distractions undermine driving safety, which is also supported by the literature (Haigney and Westerman 2001, Horrey and Wickens 2006).

There are other ways to look at covariate effects. For example, simple correlations that account for lags between different dependent variables can help visualize the time dependencies between these variables. The interrelations between multiple dependent variables can also be handled with multivariate analysis techniques, such as discriminant analysis or structural equations (Milliken and Johnson 1992, Judd et al. 1996). However, each of these techniques has different disadvantages. Simple correlations cannot account for the structure of the experimental design and one is more prone to include irrelevant variables in multivariate analyses, thus making the results harder to interpret.

This paper demonstrated the use of time-dependent covariates for a repeated measures design. This type of experimental design is very common in transportationrelated research because collecting data for multiple conditions from one subject requires less effort than recruiting one subject for each of these treatments. Another advantage is the reduction in error variance for within-subject effects. However, the time-dependent covariates can also be included in other types of experimental design.

This paper concentrated on driving simulator studies in transportation research. However, these issues also affect on-road transportation studies that employ instrumented vehicles in which more naturalistic data are collected (Reed and Green 1999, Boyce and Geller 2002). In particular, the time-dependent covariates are highly 
relevant in on-road studies, given the fact that there is typically less control in such studies than in simulator studies. More generally, as researchers consider the interplay of multiple psychological processes in describing complex adaptive behaviour, the need to consider time-dependent covariates will grow (Ehret et al. 2000, Brehmer 2005).

\section{Acknowledgments}

This research was conducted as part of the SAVE-IT program under contract by Delphi Corporation and sponsored by the National Highway Traffic Safety Administration - Office of Vehicle Safety Research. The authors acknowledge the technical support and comments provided by Mike Perel of NHTSA and Mary Stearns of the Volpe Center. Technical editing and comments were also provided by Teresa Lopes of the Public Policy Center and the students in the Human Factors and Statistical Modeling Laboratory at the University of Iowa.

\section{References}

Alm, H. and Nilsson, L., 1995, The effects of a mobile telephone task on driver behavior in a car following situation. Accident Analysis \& Prevention, 27, pp. 707-715.

Bittner, A.C., Gore, B.F. and Hooey, B.L., 1997, Meaningful assessments of simulator performance and sickness - can't have one without the other? Human Factors and Ergonomics Society 41st Annual Meeting, Albuquerque, NM (Santa Monica, CA: HFES).

Boyce, T.E. and Geller, E.S., 2002, An instrumented vehicle assessment of problem behavior and driving style: do younger males really take more risks? Accident Analysis and Prevention, 34, pp. 51-64.

Boyle, L. and Mannering, F., 2004, Impact of traveler advisory systems on driving speed: some new evidence. Transportation Research Part C, 12, pp. 57-72.

Breckenridge, R.L. and Dodd, M.O., 1991, Locus of control and alcohol placebo effects on performance in a driving simulator. Perceptual and Motor Skills, 72, pp. 751-756.

BREHMER, B., 2005, Micro-worlds and the circular relation between people and their environment. Theoretical Issues in Ergonomics Science, 6, pp. 73-93.

Broокнuis, K., 1998, How to measure driving ability under the influence of alcohol and drugs, and why. Human Psychopharmacology: Clinical and Experimental, 13, pp. 64-69.

Brouwer, W.H., Waterink, W., Van Wolffelaar, P.C. and Rothergatter, J.A., 1991, Divided attention in experienced young and older driver: lane tracking and visual analysis in a dynamic driving simulator. Human Factors, 33, pp. 573-582.

BRown, I.D., TickneR, A.H. and Simmonds, D.C.V., 1969, Interference between concurrent tasks of driving and telephoning. Journal of Applied Psychology, 53, pp. 419-424.

Chapanis, A., 1988, Some generalizations about generalizations. Human Factors, 30, pp. 253-267.

Collet, C., Petit, C., Champely, S. and Dittmar, A., 2003, Assessing workload through physiological measurements in bus drivers using automated system during docking. Human Factors, 45, pp. 539-548.

Cooper, P.J. and Zheng, Y., 2002, Turning gap acceptance decision-making: the impact of driver distraction. Journal of Safety Research, 33, pp. 321-335.

Donmez, B., Boyle, L. and Lee, J.D., 2006, The impact of distraction mitigation strategies on driving performance. Human Factors, 48, pp. 785-804.

Ehret, B.D., Gray, W.D. and Kirschenbaum, S.S., 2000, Contending with complexity: developing and using a scaled world in applied cognitive research. Human Factors, 42, pp. 8-23. 
Faucett, C.L., Schenker, N. and Elashoff, R.M., 1998, Analysis of censored survival data with intermittently observed time-dependent binary covariates. Journal of American Statistical Association, 93, pp. 427-437.

Findley, L., Fabrizio, M., Knight, H., Norcross, B.B., Laforte, A.J. and Suratt, P.M., 1989, Driving simulator performance in patients with sleep apnea. American Review of Respiratory Diseases, 140, pp. 529-530.

Grilli, L., 2005, The random effects proportional hazards model with grouped survival data: a comparison between the grouped continuous and continuation ratio versions. Journal of the Royal Statistical Society Series A, 168, p. 83.

Haigney, D. and Westerman, S.J., 2001, Mobile (cellular) phone use and driving: a critical review of research methodology. Ergonomics, 44, pp. 132-143.

Horrey, W.J. and Wickens, C.D., 2006, Examining the impact of cell phone conversations on driving using meta-analytic techniques. Human Factors, 48, pp. 196-205.

Jamson, A.H., Westerman, S.J., Hockey, G.R.J. and Carsten, O.M.J., 2004, Speech-based E-mail and driver behavior: effects of an in-vehicle message system interface. Human Factors, 46, pp. 625-639.

Judd, C.M., Mcclelland, G.H. and Smith, E.R., 1996, Testing treatment by covariate interactions when treatment varies within subjects. Psychological Methods, 1, pp. 366-378.

Khattree, R. and Naik, D.N., 1999, Applied Multivariate Statistics with SAS Software (Cary, NC: SAS Institute and Wiley).

LAm, L.T., 2002, Distractions and the risk of car crash injury: the effect of drivers' age. Journal of Safety Research, 33, pp. 411-419.

LeE, D.N., 1976, A theory of visual control of braking based on information about time to collision. Perception, 5, pp. 437-459.

Lee, J.D., Caven, B., Haake, S. and Brown, T.L., 2001, Speech-based interaction with in-vehicle computers: the effect of speech-based e-mail on drivers' attention to the road. Human Factors, 43, pp. 631-640.

Liu, L., Miyazaki, M. and Watson, B., 1999, Norms and validity of the DriVR: a virtual reality driving assessment for persons with head injuries. Cyberpsychology \& Behavior, 2, pp. 53-67.

Milliken, G.A. and Johnson, D.E., 1992, Analysis of Messy Data Volume 1: Designed Experiments (New York: Chapman \& Hall).

Rao, S., 1995, Linear Models for Multivariate Repeated Measures Data (Norfolk, Virginia: Old Dominion University).

Reed, M.P. and Green, P.A., 1999, Comparison of driving performance on-road and in a low-cost simulator using a concurrent telephone dialling task. Ergonomics, 42, pp. 1015-1037.

Rizzo, M., Mcgehee, D., Dawson, J. and Anderson, S., 2001, Simulated car crashes at intersections in drivers with Alzheimer disease. Alzheimer Disease and Associated Disorders, 15, pp. 10-20.

Schwarz, G., 1978, Estimating the dimension of a model. Annals of Statistics, 6, pp. 461-464.

Stanton, N.A., Young, M.S., Walker, G.H., Turner, H. and Randler, S., 2001, Automating the driver's control tasks. International Journal of Cognitive Ergonomics, 5, pp. 221-236.

Syzlek, J.P., Pizzimenti, C.E., Fishman, G.A., Kelich, R., Wetzel, L.C., Kagan, S. and Ho, K., 1995, A comparison of driving in older subjects with and without age-related macular degeneration. Archives of Ophthalmology, 113, pp. 1033-1040.

VAN Der LaAn, M.J. and Robins, J.M., 1998, Locally efficient estimation with current status data and time-dependent covariates. Journal of American Statistical Association, 93, pp. 693-701.

\section{About the authors}

Birsen Donmez is a PhD student and a graduate research assistant in the Mechanical and Industrial Engineering Department of the University of Iowa. She is also enrolled in the Master's programme in the Statistical and Actuarial Sciences Department. She received her MS in Mechanical and Industrial Engineering in 2004 from the University of Iowa. Her research interests include assessing human interaction with advanced technology using statistical methods. She is a recipient of Dwight David Eisenhower graduate fellowship from US Department of Transportation. 
Linda $\mathbf{~ g ~ B o y l e ~ i s ~ a n ~ a s s i s t a n t ~ p r o f e s s o r ~ i n ~ t h e ~ d e p a r t m e n t ~ o f ~ m e c h a n i c a l ~ a n d ~ i n d u s t r i a l ~ e n g i n e e r i n g ~}$ at the University of Iowa. She has a $\mathrm{PhD}$ in civil and environmental engineering from the University of Washington, and a MS and BS in industrial engineering. She teaches in the area of analytical methods for human factors engineering. Her research centres on the analysis of operator's adaptive behaviour and how individual differences influence a persons' risk of crashes and injuries.

John D. Lee is a professor in the department of mechanical and industrial engineering at the University of Iowa. He has a background in engineering and psychology, with a $\mathrm{PhD}$ in mechanical engineering from the University of Illinois at Urbana-Champaign. His research focuses on the safety and acceptance of complex human-machine systems by considering how technology mediates attention. Specific research interests include trust in technology, advanced driver assistance systems and driver distraction. 